\title{
I ntraoperative periprosthetic acetabular fractures during primary total hip arthroplasty: A case report and review of the literature
}

\author{
Xiao Xiao Zhou ${ }^{1}$, Yang Yang ${ }^{2}$, J u Liu ${ }^{1}$, Feng Gao ${ }^{1}$, Wei Yuan ${ }^{1}$, Zhengfeng Xu ${ }^{1}$, Minghui Wang ${ }^{1}$, \\ Xiuhui Wang ${ }^{1}$ \\ 1. Department of Orthopedics, Zhoupu Hospital of Pudong District, Shanghai Health Education Medical College, Shanghai, \\ China. 2. Wenzhou Medical University, Wenzhou, ZheJ iang, China
}

Correspondence: Xiao xiao ZHOU. Address: Zhoupu Hospital of Pudong, Zhouyuan Road, Pudong District 1500, Shanghai 201318, China. Email: zhouxx1493@126.com

Received: August 3, 2015

DOI : $10.5430 /$ ijdi.v3n1p19

Accepted: October 12, 2015

Online Published: October 18, 2015

\begin{abstract}
Intraoperative periprosthetic acetabular fracture (IPPAF) during primary total hip arthroplasty (pTHA) is a challenging complication because of lack of knowledge on typical intraoperative manifestation, poor recognition, and bad treatment outcomes. Intraoperative identification and proper management of fractures can decrease the failure rate of acetabular components. Intraoperative fracture manifestation has been described rarely in the literature to date. We present a case of IPPAF during PTHA, in which a functional hip was successfully saved using non-operative treatment. A review of the relevant literature is also included in this report.
\end{abstract}

\section{Keywords}

Intraoperative periprosthetic acetabular fractures, Primary total hip arthroplasty, Complication

\section{I ntroduction}

Periprosthetic fracture is a challenging complication of total hip arthroplasty (THA). Although reports on intraoperative periprosthetic femoral fractures (IPPFFs) after THA have increased ${ }^{[1-3]}$, intraoperative periprosthetic acetabular fractures (IPPAFs) are relatively uncommon ${ }^{[4-7]}$. IPPAF management is difficult and usually leads to early failure ${ }^{[6]}$. Moreover, patients who undergo THA revision because of periprosthetic fracture have poorer functional outcomes and higher death rates than those who undergo THA revision for aseptic loosening ${ }^{[8]}$.

The clinical features of these fractures have received only little attention in the literature because of the limited information on typical clinical manifestations. Most cases of intraoperative fractures lose the best chance of early treatment, which leads to revision of the acetabular cup. IPPAFs may occur during component removal in a revision. This situation is more complicated and difficult to manage compared with primary total hip arthroplasty (pTHA) and is usually accompanied by serious osteolysis and massive loss of acetabular bone stock. Such a case has been explained previously ${ }^{[9]}$, and is beyond the scope of this review. 
Our study presents the case of an elderly woman with IPPAF, whose typical intraoperative symptoms started during the patient's pTHA. Non-operative treatment was used to save the functional hip. This article describes the case and provides a review of the literature.

\section{Case report}

A 73-year-old woman with a weight of $40 \mathrm{~kg}$ and a height of $1.54 \mathrm{~m}$ underwent pTHA in June 2012 following right subcapital femoral neck fracture, which was caused by an accident.

Three days after admission, the patient underwent pTHA. The patient was positioned and stabilized on the operating table in the lateral decubitus position. A posterolateral approach to the hip was performed. The operating table and the bony anatomy landmarkers were used as reference in the prostheses implantation. Two 2.5-mm Kirschner wires were fixed into the acetabular bone to help expose the acetabulum. One of the two Kirschner wires was too close to the retrolateral wall of the acetabulum (see Figure 1), causing a split line. A Trilogy AB ${ }^{\text {тм }}$ (Alternate Bearing) Acetabular System (Zimmer, Inc., USA), which includes cementless alumina/alumina ceramic bearing couples with femoral stem size (12\#) coated with hydroxyapatite on the proximal third, an alumina ceramic head with a 28-mm diameter, and an alumina ceramic insert (liner) with a metal acetabular hemispherical porous cup (46 mm), was inserted. The intended cup position was at $40^{\circ}$ inclination and $25^{\circ}$ anteversion. A 2-mm press-fit was used for the cementless socket fixation. When the cup was impacted to the bone bed, it moved with the whole pelvis when the stem was shaken, which showed that the cup was stable. The size and neck length of the prostheses were selected according to the correct limb-length that corresponded with gluteal muscle tension. The external rotators were reattached to the greater trochanter using non-absorbable sutures.

Figure 1. Schematic indicating how the Kirschner wires were fixed into the acetabular bone on a lateral image of a pelvic specimen. The line A represented the Kirschner wire fixed into the retrolateral wall of the acetabulum, the line B was fixed into the anterolateral wall, and the dash line $\mathrm{C}$ represented split line of retrolateral wall in the acetabulum

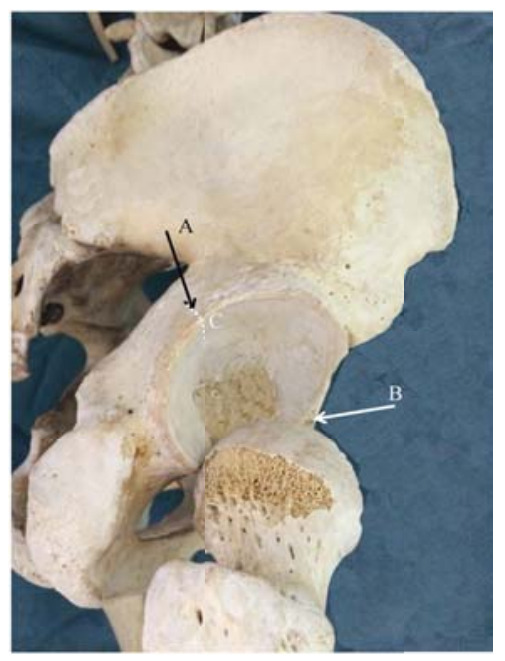

The postoperative lateral hip radiograph of the posterior column revealed an IPPAF, which was not observed on the anteroposterior (AP) pelvic radiograph. The patient was scheduled for follow up and ordered to stay in bed for 12 weeks until fracture union was achieved (see Figures 2, 3). Then, the patient was given weight bear until 7 months after the operation (see Figure 4). The patient was followed up for two years, she lived a near-normal life, and the two years postoperative AP radiograph show that the prostheses appears stable, and no sink or lucent line is observed (see Figure 5).

The intraoperative and postoperative findings are as follows:

(1) The insertion position was inconsistent with the last reamer. The acetabular component location was changed, which indicated mild sinking of the acetabular component, but the acetabular component remained well-fixed with an excellent press-fit, and good anteversion and inclination were retained (see Figure 2A). 
(2) Mild increase bleeding instead of hemostasis was observed. When the reaming of the bony acetabulum is completed and the subchondral cancellous bone is exposed, normally a good press-fit between the component and the cancellous bone will stop the bleeding. However, with IPPAFs, a mild increase in bleeding can be found after acetabular component insertion.

(3) A mild fracture line appeared on the acetabular rim around the Kirschner wire was observed upon impaction of the acetabular component. Without intraoperative X-ray or further examination, the fracture line was considered only as a split of the of poster wall, not a fracture of the posterior column.

(4) An undisplaced acetabular fracture in the posterior column was observed in the postoperative lateral hip radiograph (see Figure 2B), but not on the AP pelvic radiograph (see Figure 2A).

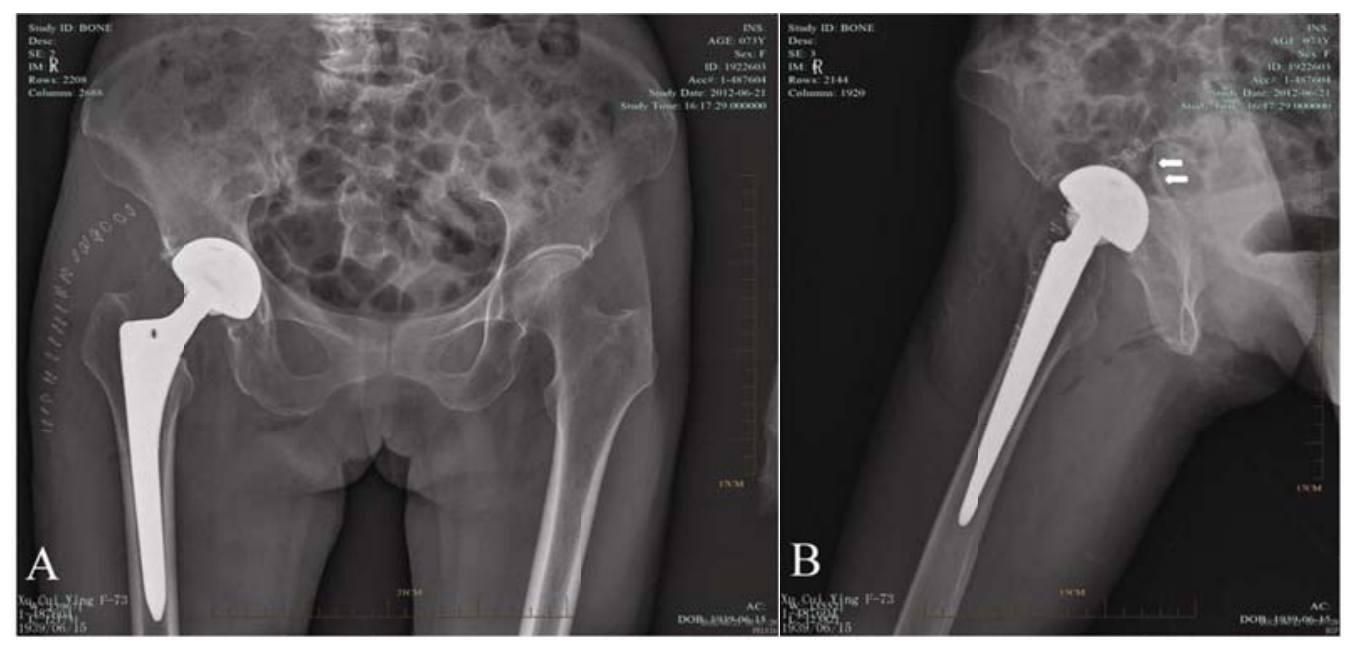

Figure 2. A. The immediate postoperative AP radiograph does not show any signs of fracture; B. Lateral radiograph reveals the fracture of the posterior column (arrows show fracture line)
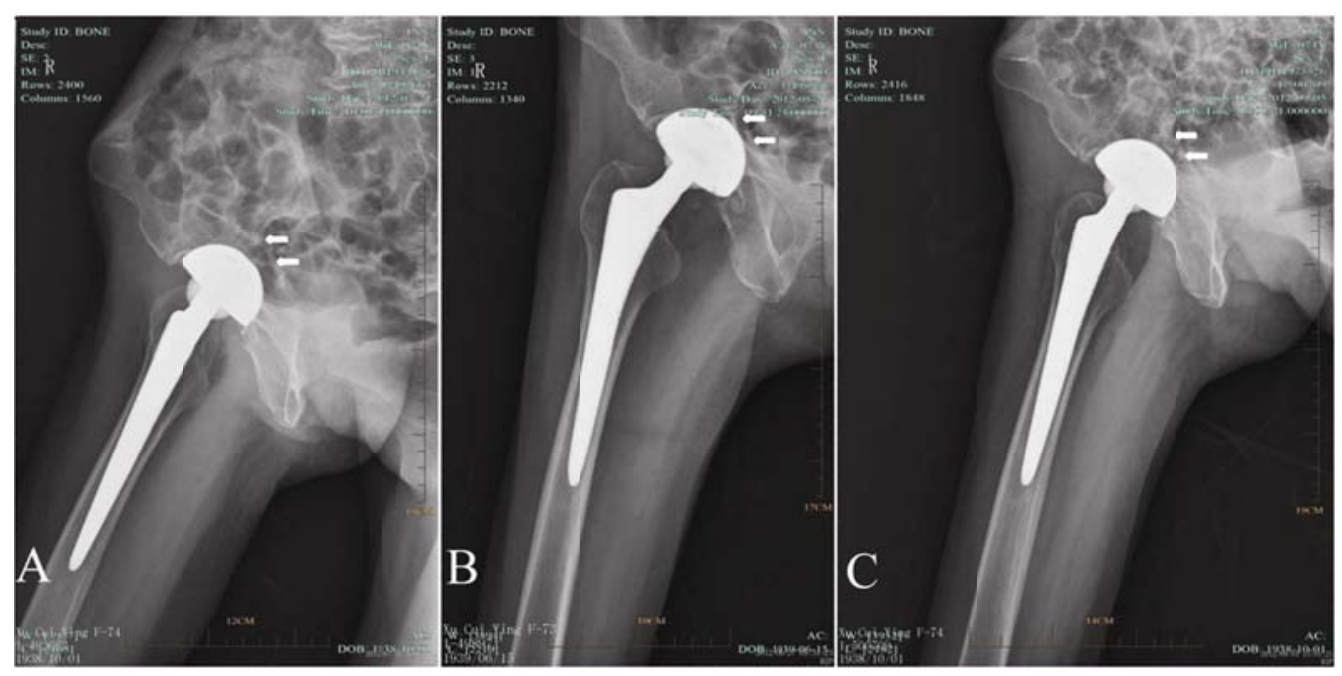

Figure 3. A. Four weeks after surgery, radiographs demonstrate no changes in the fracture; B. Eight weeks postoperatively, no prominent osseous growth can be observed in the fracture; C. Twelve weeks after surgery, radiographs demonstrates prominent osseous ingrowth and union of the fracture (arrows show fracture line) 


\section{Discussion}

\subsection{Epidemiology}

IPPAF is a rare complication of pTHA. No reports have been found on IPPAFs during cemented pTHA until now because they are more commonly associated with uncemented cups ${ }^{[6,10-13]}$. Several fractures found after pTHA may have developed from unrecognized intraoperative fractures ${ }^{[14]}$. Mild fractures are difficult to identify because they can be asymptomatic.

Figure 4. A. The seventh month postoperative AP radiograph does not show any signs of fracture. At fourth month postoperative, the patient's weight bearing in the prostheses is stable and does not have any lucent line. The patient reported no pain or discomfort; B. Seven months after surgery, lateral radiograph reveals the fracture of the posterior column osseous ingrowth and union. The prostheses appears stable and no sink or lucent line (arrows show fracture line obscure) is observed

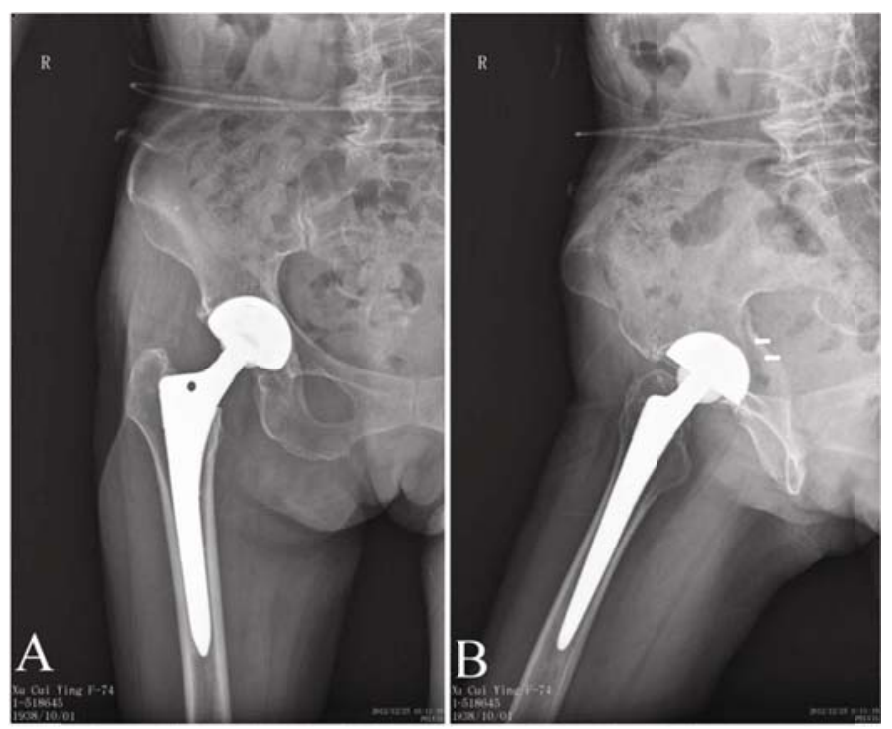

Figure 5. The two years postoperative AP radiograph show that the prostheses appears stable, and no sink or lucent line is observed

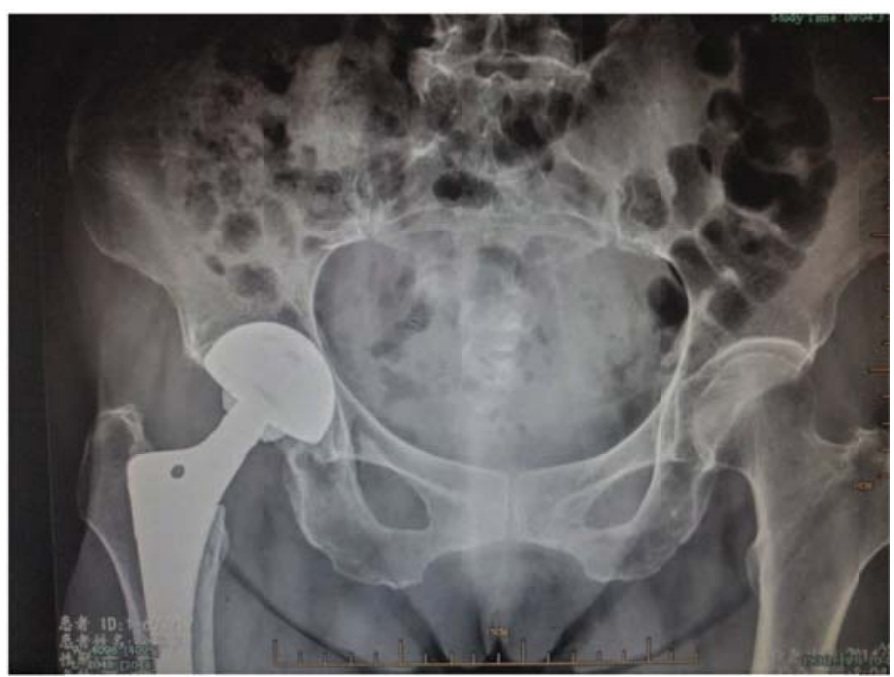

A four-case series presenting the results of IPPAFs during PTHA in patients have been published in the literature ${ }^{[14-17]}$, the epidemiological characteristics of which are summarized in Tables 1 and 2.

A previous study reported that IPPAF occurrence rates during cemented and uncemented pTHA were 0 and 0.04\%, respectively ${ }^{[16]}$, which confirms that IPPAF is an uncommon complication. However, another study revealed that the IPPAF rate during PTHA was at 3.4\% (11 of 326) ${ }^{[17]}$. Maybe the rate of the IPPAF rate during pTHA was higher than consided as numerous patients were misdiagnosed. 


\subsection{Risk factors}

Several risk factorsthat can lead to IPPAFs during cementless acetabular component fixation are as follows: a) impaction of cup with excessive force; b) use of oversized cup ( $>2 \mathrm{~mm}$ ) for press-fit ${ }^{[15]}$; c) osteoporosis or osteopenia ${ }^{[14,15,18-20] \text {; }}$ d) inconsistency of the insertion spatial location of the cup component with the reamed area; e) excessive reaming, resulting in cancellous bone loss, exposure of the inner lamellar bone, and lack of cancellous bone buffer upon cup insertion; f) incision exposures, such as hooks ${ }^{[17]}$, wherein the Kirschner wire used to expose the incision or split line may potentially induce and increase the fracture; and g) other reasons, such as elliptical monoblock cups ${ }^{[14,15]}$. Considering the lack of experience, these signs tend to be ignored, and the best opportunity to manage fractures intraoperatively is lost.

\subsection{Clinical symptoms and intraoperative manifestations}

Clinical symptoms include mild trochanteric discomfort, mild to severe hip pain, and significant groin pain (see Table 2). However, these symptoms are specific to postoperative IPPAFs. In these cases, a few methods can be used to address these problems.

There were no documents about intraoperative manifestations of IPPAFs. Meanwhile, intraoperative identification and appropriate treatment of IPPAFs can decrease the failure rate. Thus, intraoperative identification of IPPAFs is important.

\subsection{Classification}

Several classifications for IPPAFs can be used. The four factors that should be considered are as follows: a) component stability, b) fracture stability and displacement or otherwise, c) anatomic location of fractures, and d) intraoperative or postoperative identification.

The Vancouver classification system of periprosthetic fractures can be used in the assessment and management of IPPAFs in pTHA ${ }^{[21]}$. The classification scheme includes three types. Type I represents an undisplaced fracture that does not compromise the stability of the component; type II indicates an undisplaced fracture that potentially compromises the stability of the reconstruction, such as a transverse fracture of the acetabulum or an oblique fracture that separates the anterior column and dome from the posterior column; and type III corresponds to a displaced fracture. A drawback of the Vancouver classification system is the non-consideration of the time element, i.e., whether the fracture is confirmed intraoperatively or postoperatively.

Another IPPAF classification system based on anatomic location was designed by Della Valle CJ, et al. ${ }^{[22]}$ and Callaghan, et al. ${ }^{[23]}$.

\subsection{Treatment}

The principles of treatment include fracture stabilization to provide sufficient initial stability of the cup component and, ultimately, the achievement of fracture union and maximized hip function ${ }^{[20,23]}$. Some recommendations for IPPAF treatment are available ${ }^{[21]}$.

When IPPAF is diagnosed intraoperatively and the patient has an undisplaced fracture with a stable component, the prosthesis can be left in situ, and standard THA rehabilitation protocols can be applied. If the component is unstable, fracture stability should be considered. In the case of a stable and strong fracture which can provide sufficient initial stability for the cup component, acetabular re-reaming can be performed and a suitable acetabular component can be selected to re-obtain press-fit. Alternatively, a larger component exchange may be needed ${ }^{[17]}$, along with assorted supplemental screw fixation. If the fracture is unstable and not strong enough to provide initial stability for the cup component and when substantial motion is evident at the fracture site with the presence of pelvic dissociation, reduction and internal fixation with a pelvic reconstruction plate may be performed. After the pelvis is stabilized, the acetabulum may be reconstructed with standard techniques. 
When an undisplaced IPPAF is diagnosed postoperatively, and the fracture potentially compromises the stability of the reconstruction, decision making regarding these conditions becomes more difficult. Similar to the case presented in this paper, patients can be instructed to keep the operative limb non-weight bearing for 6 weeks to 8 weeks.

Component stability should be carefully determined with the use of intraoperative stress testing of the pelvis and the acetabular component, which may require cup removal to fully examine the acetabulum. Givern that innitial postoperative radiographs did not clearly reveal the fractures ${ }^{[15]}$. The ilium oblique view and obturator oblique view of the radiographs were recommended, particularly for patients with suspected fractures. CT scan should also be used if necessary.

\section{Conclusion}

Firm conclusions cannot be made based on this study because of the small retrospective cohort, and limited documents and patients.

Although IPPAFs are difficult to identify, heightened alertness for suspicion must be maintained. And IPPAF prevention is more important than fracture management itself. Many fractures can be prevented with careful technique ${ }^{\text {[24] }}$.

\section{References}

[1] Bethea JS, De Andrade JR, Fleming LL, et al. Proximal femoral fractures following total hip arthroplasty. Clin Orthop. 1982; 170: 95-106. http://dx.doi.org/10.1097/00003086-198210000-00013

[2] Johansson JE, McBroom R, Barrington TW, et al. Fracture of the ipsilateral femur in patients with total hip replacement. J. Bone and Joint Surg. 1981; 63-A: 1435-42.

[3] Scott RD, Turner RH, Leitzes SM, et al. Femoral fractures in conjunction with total hip replacement. J Bone and Joint Surg. 1975; 57-A: 494-501.

[4] Harvie P, Gundle R, Willett K. Traumatic periprosthetic acetabular fracture: life threatening haemorrhage and a novel method of acetabular reconstruction. Injury. 2004; 35: 819-22. http://dx.doi.org/10.1016/S0020-1383(03)00218-3

[5] Miller AJ. Late fracture of the acetabulum after total hip replacement. J Bone Joint Surg [Br]. 1972; 54: 600-6. PMid:4639435

[6] Peterson CA, Lewallen DG. Periprosthetic fracture of the acetabulum after total hip arthroplasty. J Bone Joint Surg [Am]. 1996; 78: 1206-13. PMid:8753713

[7] Chatoo M, Parfitt J, Pearse MF. Periprosthetic acetabular fracture associated with extensive osteolysis. J Arthroplasty. 1998; 13: 843-5. http://dx.doi.org/10.1016/S0883-5403(98)90042-0

[8] Young SW, Walker CG, Pitto RP. Functional outcome of femoral peri prosthetic fracture and revision hip arthroplasty: a matched-pair study from the New Zealand Registry. Acta Orthop. 2008; 79: 483-8. PMid:18766480 http://dx.doi.org/10.1080/17453670710015463

[9] Davidson D, Pike J, Garbuz D, et al. Intraoperative periprosthetic fractures during total hip arthroplasty. Evaluation and management. J Bone Joint Surg [Am]. 2008; 90(9): 2000-12. PMid:18762663 http://dx.doi.org/10.2106/JBJS.H.00331

[10] Adler E, Stuchin SA, Kummer FJ. Stability of press-fit acetabular cups. J Arthroplasty. 1992; 7: 295-301. http://dx.doi.org/10.1016/0883-5403(92)90052-R

[11] Curtis MJ, Jinnah RH, Wilson VD, et al. The initial stability of uncemented acetabular components. J Bone Joint Surg [Br]. 1992; 74: 372-6. PMid:1587880

[12] MacKenzie JR, Callaghan JJ, Pedersen DR, et al. Areas of contact and extent of gaps with implantation of oversized acetabular components in total hip arthroplasty. Clin Orthop Relat Res. 1994; 298: 127-36. http://dx.doi.org/10.1097/00003086-199401000-00018

[13] Stiehl JB, MacMillan E, Skrade DA. Mechanical stability of porous-coated acetabular components in total hip arthroplasty. J Arthroplasty. 1991; 6: 295-300. http://dx.doi.org/10.1016/S0883-5403(06)80179-8

[14] Desai G, Ries MD. Early postoperative acetabular discontinuity after total hip arthroplasty. J Arthroplasty. 2011; 26(8): 1570.e17-9.

[15] Sharkey PF, Hozack WJ, Callaghan JJ, et al. Acetabular fracture associated with cementless acetabular component insertion: a report of 13 cases. J Arthroplasty. 1999; 14: 426-31. http://dx.doi.org/10.1016/S0883-5403(99)90097-9 
[16] Haidukewych GJ, Jacofsky DJ, Hanssen AD, et al. Intraoperative fractures of the acetabulum during primary total hip arthroplasty. J Bone Joint Surg Am. 2006; 88: 1952-6. PMid:16951110 http://dx.doi.org/10.2106/JBJS.E.00890

[17] He ZY, Di ZL, Zhang JH, et al. Strategy of prevention and management of intraoperative fractures of acetabulum during primary total hip arthroplasty. Zhongguo Gu Shang (in Chinese). 2009; 22(2): 81-3. PMid:19281003

[18] Berry DJ. Management of periprosthetic fractures: the hip. J Arthroplasty. 2002, 17(4 Suppl 1): 11-3. PMid:12068395 http://dx.doi.org/10.1054/arth.2002.32682

[19] Gras F, Marintschev I, Klos K, et al. Navigated percutaneous screw fixation of a periprosthetic acetabular fracture. J Arthroplasty. 2010; 25(7): 1169. e1-4.

[20] Meek RM, Norwood T, Smith R, et al. The risk of peri-prosthetic fracture after primary and revision total hip and knee replacement. J Bone Joint Surg [Br]. 2011; 93(1): 96-101. PMid:21196551 http://dx.doi.org/10.1302/0301-620X.93B1.25087

[21] Duncan CP, Masri BA. Fractures of the femur after hip replacement. Instr Course Lect. 1995; 44: 293-304. PMid:7797866

[22] Della Valle CJ, Momberger NG, Paprosky WG. Periprosthetic fractures of the acetabulum associated with a total hip arthroplasty. Instr Course Lect. 2003; 52: 281-90. PMid:12690856

[23] Callaghan JJ. Periprosthetic fractures of the acetabulum during and following total hip arthroplasty. Instr Course Lect. 1998; 47: 231-5. PMid:9571423

[24] Mitchell PA, Greidanus NV, Masri BA, et al. The prevention of periprosthetic fractures of the femur during and after total hip arthroplasty. Instr Course Lect. 2003; 52: 301-8. PMid:12690858 